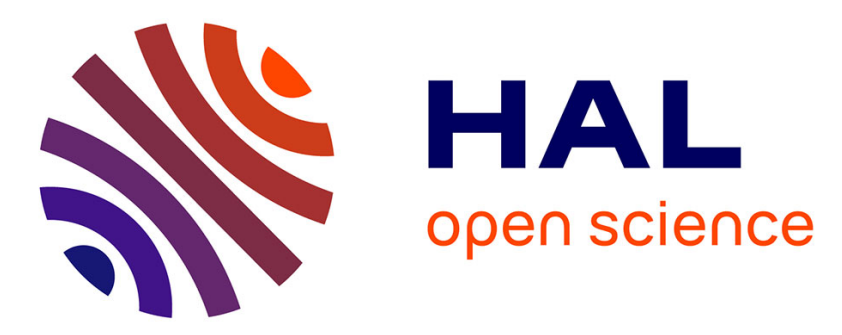

\title{
A construction of a $\beta$-coalescent via the pruning of Binary Trees
}

\author{
Romain Abraham, Jean-François Delmas
}

\section{To cite this version:}

Romain Abraham, Jean-François Delmas. A construction of a $\beta$-coalescent via the pruning of Binary Trees. Journal of Applied Probability, 2013, 50 (3), pp.772-790. hal-00711518v2

\section{HAL Id: hal-00711518 \\ https://hal.science/hal-00711518v2}

Submitted on 9 Nov 2012

HAL is a multi-disciplinary open access archive for the deposit and dissemination of scientific research documents, whether they are published or not. The documents may come from teaching and research institutions in France or abroad, or from public or private research centers.
L'archive ouverte pluridisciplinaire HAL, est destinée au dépôt et à la diffusion de documents scientifiques de niveau recherche, publiés ou non, émanant des établissements d'enseignement et de recherche français ou étrangers, des laboratoires publics ou privés. 


\title{
A CONSTRUCTION OF A $\beta$-COALESCENT VIA THE PRUNING OF BINARY TREES
}

\author{
ROMAIN ABRAHAM AND JEAN-FRANÇOIS DELMAS
}

\begin{abstract}
Considering a random binary tree with $n$ labelled leaves, we use a pruning procedure on this tree in order to construct a $\beta\left(\frac{3}{2}, \frac{1}{2}\right)$-coalescent process. We also use the continuous analogue of this construction, i.e. a pruning procedure on Aldous's continuum random tree, to construct a continuous state space process that has the same structure as the $\beta$-coalescent process up to some time change. These two constructions enable us to obtain results on the coalescent process such as the asymptotics on the number of coalescent events or the law of the blocks involved in the last coalescent event.
\end{abstract}

\section{INTRODUCTION}

Let $\Lambda$ be a finite measure on $[0,1]$. A $\Lambda$-coalescent $(\Pi(t), t \geq 0)$ is a Markov process which takes values in the set of partitions of $\mathbb{N}^{*}=\{1,2, \ldots\}$ introduced in [25]. It is defined via the transition rates of its restriction $\left(\Pi^{[n]}(t), t \geq 0\right)$ to the $n$ first integers: if $\Pi^{[n]}(t)$ is composed of $b$ blocks, then $k(2 \leq k \leq b)$ fixed blocks coalesce at rate:

$$
\lambda_{b, k}=\int_{0}^{1} u^{k-2}(1-u)^{b-k} \Lambda(d u)
$$

In particular a coalescent event arrives at rate:

$$
\lambda_{b}=\sum_{k=2}^{b}\left(\begin{array}{l}
b \\
k
\end{array}\right) \lambda_{b, k} .
$$

As examples of $\Lambda$-coalescents, let us cite Kingman's coalescent $\left(\Lambda(d x)=\delta_{0}(d x)\right.$, see [23]), the Bolthausen-Sznitman coalescent $\left(\Lambda(d x)=\mathbf{1}_{(0,1)}(x) d x\right.$, see [13]), or $\beta$-coalescents $(\Lambda(d x)$ is the $\beta(2-\alpha, \alpha)$ distribution with $0<\alpha<2$, see $[12,10]$, or the $\beta(2-\alpha, \alpha-1)$ distribution with $1<\alpha<2$, see [17]). We refer to the survey [9] for further results on coalescent processes.

The goal of this paper is to give a new representation for the $\beta(3 / 2,1 / 2)$-coalescent using the pruning of random binary trees. This kind of idea has already been used in [18] where the Bolthausen-Sznitman coalescent is constructed via the cutting of a random recursive tree.

1.1. Pruning of binary trees. Now we describe the coalescent associated with the pruning of the random binary tree.

We recall the normalization constant in the beta distribution for $a>0, b>0$ :

$$
\beta(a, b)=\frac{\Gamma(a) \Gamma(b)}{\Gamma(a+b)}=\int_{(0,1)} x^{a-1}(1-x)^{b-1} d x .
$$

Fix an integer $n$ and consider a uniform random ordered binary tree with $n$ leaves $(2 n-1$ vertices plus a root; $2 n-1$ edges). Label these leaves from 1 to $n$ uniformly at random. After

Date: November 9, 2012.

This work is partially supported by the "Agence Nationale de la Recherche", ANR-08-BLAN-0190. 
an exponential time of parameter:

$$
\lambda_{n}=(n-1) \beta\left(\frac{1}{2}, n-\frac{1}{2}\right),
$$

choose one of the $n-1$ inner vertices of the tree uniformly at random, coalesce all the leaves of the subtree attached at the chosen node and remove that subtree from the original tree. Restart then the process with the resulting tree (using $\lambda_{k}$ as the new parameter of time, where $k$ is the new number of leaves) until all the leaves coalesce into a single one.

Figure 1 gives an example of such a coalescence for $n=5$.
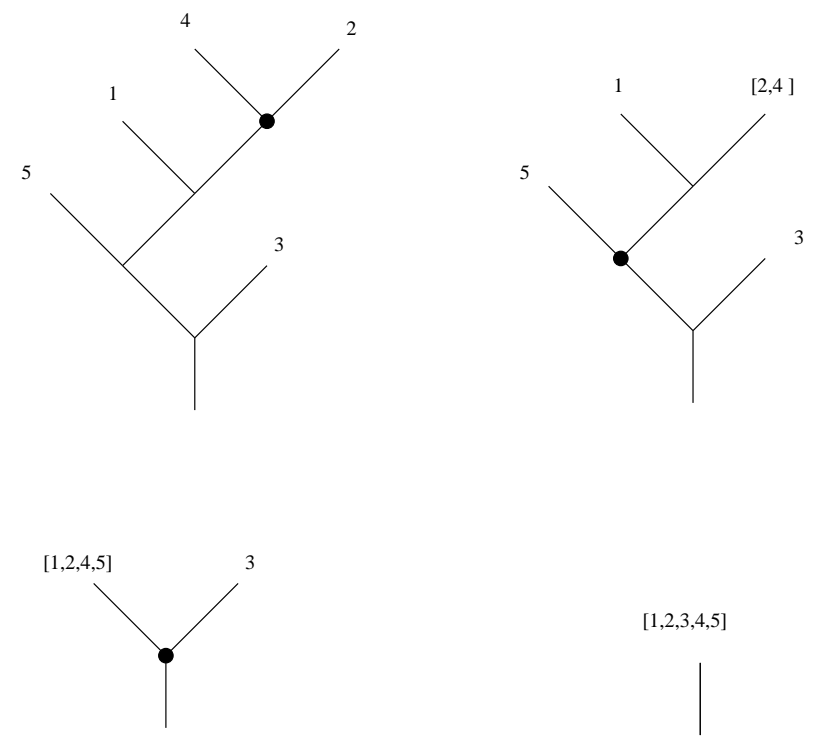

$[1,2,3,4,5]$

Figure 1. An example of the coalescence of a binary tree for $n=5$. The selected node is in bold and the times between each picture are exponentially distributed with respective parameter $\lambda_{5}, \lambda_{4}, \lambda_{2}$.

This defines a process $\left(\Pi^{[n]}(t), t \geq 0\right)$. The main result of the paper is the following Theorem.

Theorem 1.1. The process $\left(\Pi^{[n]}(t), t \geq 0\right)$ defined as the coalescent associated with the pruning of the random binary tree is the restriction to $\{1, \ldots, n\}$ of a $\beta(3 / 2,1 / 2)$-coalescent with coalescent measure:

$$
\Lambda(d u)=\sqrt{\frac{u}{1-u}} d u .
$$

Let us remark that the $\beta$-coalescents introduced in [12] and usually studied are $\beta(2-\alpha, \alpha)$ coalescents with $1<\alpha<2$. Here we have $\alpha=1 / 2$ and this does not enter the usual case. The reason is that for $1<\alpha<2$, the $\beta$-coalescent comes down from infinity $(\Pi(t)$ has a.s. a finite number of blocks for any $t>0$ ) which is not the case for $\alpha=1 / 2$ according to the criterion from [9] Theorem 3.5 or [25] as:

$$
\int_{0}^{1} \frac{\Lambda(d u)}{u}<+\infty
$$


1.2. Pruning of Aldous's CRT. A coalescent process may also be viewed as a process $(\mathcal{I}(t), t \geq 0)$ taking values in the interval partitions of $[0,1]$. The length of each interval represents the mass of a block, and the process represents blocks (whose sizes sum to 1 ) that merge together as time passes. We can go from this interval partition-valued process to the previous $\Lambda$-coalescent framework by the classical paintbox procedure (see for instance [26]): consider $\left(U_{i}\right)_{i \in \mathbb{N}}$ a sequence of i.i.d. uniform random variables on $[0,1]$, independent of the process $\mathcal{I}$ and, for every $t \geq 0$, say that the integers $i$ and $j$ belong to the same block in $\Pi(t)$ if and only if $U_{i}$ and $U_{j}$ belong to the same interval in $\mathcal{I}(t)$.

The continuous analogue of the binary tree is Aldous's continuum random tree (CRT) which can be obtained as the limit (in an appropriate sense) of the rescaled uniform binary tree with $n$ leaves when the number of leaves $n$ tends to infinity. A pruning theory of such a continuum tree has been introduced in [5] (see [4] for a general theory of the pruning of Lévy trees) and will be recalled in Section 3.1. Using this pruning procedure, we are able to define an interval-partition-valued process in Section 3.2 which has the same structure as the $\beta(3 / 2,1 / 2)$-coalescent except for the times (when sampling $n$ points uniformly distributed on $(0,1)$, the time interval between two coalescences is not exponentially distributed). However, we conjecture that an appropriate change of time would be enough so that the intervalpartition-valued process is really associated with the $\beta(3 / 2,1 / 2)$-coalescent via the paintbox procedure. This gives a nice interpretation of the dust (or fraction of singletons) in the $\beta(3 / 2,1 / 2)$-coalescent.

1.3. Number of coalescent events and last coalescent event. The construction using discrete trees allows us to recover in Section 4.1 the asymptotic distribution of the number of coalescent events given by [19] in a more general framework, see also [21].

Proposition 1.2. Let $X_{n}^{\prime}$ be the number of collisions undergone by $\left(\Pi^{[n]}(t), t \geq 0\right)$. Then we have:

$$
\frac{X_{n}^{\prime}}{\sqrt{n}} \underset{n \rightarrow \infty}{\stackrel{(d)}{\longrightarrow}} \sqrt{2} Z
$$

where $Z$ has a Rayleigh distribution with density $x \mathrm{e}^{-x^{2} / 2} \mathbf{1}_{\{x>0\}}$.

Let us remark that according to Section 5.4 in [19] $Z$ is distributed as:

$$
\frac{2}{\sqrt{\pi}} \int_{0}^{\infty} d t \mathrm{e}^{-S_{t} / 2}
$$

where $\left(S_{t}, t \geq 0\right)$ is a subordinator with $\mathbb{E}\left[\exp \left(-\lambda S_{t}\right)\right]=\exp (-t \Phi(\lambda))$ where for $\lambda>0$ :

$\Phi(\lambda)=\int_{0}^{1}\left(1-(1-u)^{\lambda}\right) \frac{\Lambda(d u)}{u^{2}}=\int_{0}^{1}\left(1-(1-u)^{\lambda}\right) u^{-3 / 2}(1-u)^{-1 / 2} d u=2 \sqrt{\pi} \frac{\Gamma\left(\lambda+\frac{1}{2}\right)}{\Gamma(\lambda)}$.

(See [11] for more results on exponentials of Lévy processes.)

The continuum tree construction allows us to study the last coalescent event (see [18] for similar questions for the Bolthausen-Sznitman coalescent, and [14] for stationary CSBP). In Section 4.2, we consider the number $E_{n}$ of external branches or singletons involved in the last coalescent event as well as the number of blocks $B_{n}$ involved in the last coalescent event.

Proposition 1.3. Let $B_{n}$ be the number of blocks and $E_{n}$ be the number of singletons involved in the last coalescent event of $\left(\Pi^{[n]}(t), t \geq 0\right)$. Then we have:

$$
\left(B_{n}, E_{n}\right) \underset{n \rightarrow \infty}{\stackrel{(d)}{\longrightarrow}}(B, E)
$$


where $(B-E, E)$ are finite random variables with generating function $\Phi$ given, for $\rho, \rho_{*} \in[0,1]$ by:

$$
\begin{aligned}
\Phi\left(\rho, \rho_{*}\right) & =\mathbb{E}\left[\rho^{B-E} \rho_{*}^{E}\right] \\
& =\rho\left(1+\log (2)-\log \left(1+\sqrt{1-\rho_{*}}-\frac{\rho+\rho_{*}}{2}\right)\right)-\sqrt{\rho} \log \left(\frac{1+\sqrt{1-\rho_{*}}+\sqrt{\rho}}{1+\sqrt{1-\rho_{*}}-\sqrt{\rho}}\right) .
\end{aligned}
$$

Furthermore $B-E$ is stochastically less than (or equal to) $E+1$.

Notice that $\Phi(1,1)=1$ which indeed implies that $B$ and $E$ are finite, that is $B_{n}$ and $E_{n}$ are of order 1 . However, $B-E$ and $E$ have infinite expectation, as this can be checked from their generating functions given below.

The generating function of $E$ is given by:

$$
\mathbb{E}\left[\rho_{*}^{E}\right]=\Phi\left(1, \rho_{*}\right)=1-2 \log \left(1+\frac{\sqrt{1-\rho_{*}}}{2}\right),
$$

and the generating function of $B-E$ is given by:

$$
\mathbb{E}\left[\rho^{B-E}\right]=\Phi(\rho, 1)=\rho(1+\log (4)-\log (1-\rho))-\sqrt{\rho} \log \left(\frac{1+\sqrt{\rho}}{1-\sqrt{\rho}}\right) .
$$

There is a nice interpretation of the distribution of $B-E-1$ given after Proposition 4.2. The generating function of $B$ is given by:

$$
\mathbb{E}\left[\rho^{B}\right]=\Phi(\rho, \rho)=\rho(1+\log (2)-\log (1+\sqrt{1-\rho}-\rho))-\sqrt{\rho} \log \left(\frac{1+\sqrt{1-\rho}+\sqrt{\rho}}{1+\sqrt{1-\rho}-\sqrt{\rho}}\right) .
$$

Of course we have a.s. $B \geq 2$.

Remark 1.4. We can compute various quantities related to $E$ and $B$. We have:

$$
\begin{aligned}
& \mathbb{P}(E=0)=1-2 \log \left(\frac{3}{2}\right) \simeq 0.19, \quad \mathbb{P}(E=1)=\frac{1}{3}, \quad \mathbb{P}(E=2)=\frac{1}{9}, \\
& \mathbb{P}(B-E=0)=0, \quad \mathbb{P}(B-E=1)=\log (4)-1 \simeq 0.39, \quad \mathbb{P}(B-E=2)=\frac{1}{3}, \\
& \mathbb{P}(B=0)=\mathbb{P}(B=1)=0, \quad \mathbb{P}(B=2)=\frac{5}{12}, \quad \mathbb{P}(E=3)=\frac{23}{160} .
\end{aligned}
$$

In particular, we have $\mathbb{P}(E>5) \leq 25 \%, \mathbb{P}(B>5) \leq 32 \%$ and $\mathbb{P}(B-E>5) \leq 11 \%$.

The paper is organized as follows. The proof of Theorem 1.1 is given in Section 2. The link with Aldous' CRT, presented in Section 3.1, is given in Section 3.2 using a pruning procedure; the reduced sub-trees are presented in Section 3.3. A proof and a comment on Proposition 1.2 are given in Section 4.1. Proposition 1.3 is proved in Sections 4.2.2 and 5.

\section{The $\beta(3 / 2,1 / 2)$-COALESCENT}

In order to prove Theorem 1.1, we first need to compute the rates $\lambda_{n, k}$ at which $k$ given blocks among $n$ blocks coalesce. This is the purpose of the next Proposition.

Proposition 2.1. For the coalescent of the random binary tree, we have for any $2 \leq k \leq n$,

$$
\lambda_{n, k}=\beta\left(k-\frac{1}{2}, n-k+\frac{1}{2}\right) .
$$


We recall the duplication formula for $a>0$ :

$$
\Gamma\left(a+\frac{1}{2}\right)=\frac{\sqrt{\pi}}{2^{2 a-1}} \frac{\Gamma(2 a)}{\Gamma(a)} .
$$

Proof. Let us first remark that, by construction, since there are $n-1$ internal vertices, we have:

$$
\lambda_{n, n}=\frac{\lambda_{n}}{n-1}=\beta\left(n-\frac{1}{2}, \frac{1}{2}\right)=\sqrt{\pi} \frac{\Gamma\left(n-\frac{1}{2}\right)}{\Gamma(n)} .
$$

It is well known that the number of ordered binary trees with $n$ leaves is given by the Catalan numbers:

$$
b_{n}=\frac{1}{n}\left(\begin{array}{c}
2 n-2 \\
n-1
\end{array}\right)=\frac{(2 n-2) !}{(n-1) ! n !} .
$$

Hence the number of ordered binary trees with $n$ labelled leaves is:

$$
C_{n}=n ! b_{n}=\frac{(2 n-2) !}{(n-1) !}=\frac{2^{2 n-2}}{\sqrt{\pi}} \Gamma\left(n-\frac{1}{2}\right) .
$$

Consider a binary tree with $n$ labelled leaves. Let $2 \leq k \leq n$. Fix $k$ labels, say the $k$ first. For these labels to coalesce at the same time, the leaves with these $k$ labels must exactly lie in a single subtree of the initial tree. Therefore, to construct such a tree, we must choose an ordered binary tree with $k$ leaves labelled from 1 to $k$ ( $C_{k}$ possibilities), choose an ordered binary tree with $n-k+1$ leaves labelled from $k$ to $n\left(C_{n-k+1}\right.$ possibilities $)$ and graft the tree with $k$ leaves at the leaf labelled $k$. Then, for the $k$ first labels to coalesce, the chosen branch must be the branch that links the two subtrees (and each branch is cut at rate $\lambda_{n, n}$ ). Therefore, we have:

$$
\begin{aligned}
\lambda_{n, k} & =\frac{C_{k} C_{n-k+1}}{C_{n}} \lambda_{n, n} \\
& =\frac{2^{2 k-2}}{\sqrt{\pi}} \Gamma\left(k-\frac{1}{2}\right) \frac{2^{2 n-2 k}}{\sqrt{\pi}} \Gamma\left(n-k+\frac{1}{2}\right) \frac{\sqrt{\pi}}{2^{2 n-2}} \frac{1}{\Gamma\left(n-\frac{1}{2}\right)} \sqrt{\pi} \frac{\Gamma\left(n-\frac{1}{2}\right)}{\Gamma(n)} \\
& =\beta\left(k-\frac{1}{2}, n-k+\frac{1}{2}\right) .
\end{aligned}
$$

This proves that the process evolves like a $\Lambda$-coalescent with $\Lambda$ given by (4) up to the time of the first merger. To finish the proof of Theorem 1.1, it remains to prove that, after that first merger, the resulting tree is still a uniform binary tree with uniform labeled leaves.

Let us fix $k \leq n$ and let $T_{k}$ be a tree with $k$ labelled leaves, one being labelled by the block $\left[i_{1}, \ldots, i_{n-k+1}\right]$, the others being labelled by singletons. We want to compute the probability of obtaining that tree after the first merger. From this tree, we construct a tree with $n$ leaves by grafting, on the leaf of $T_{k}$ labelled by the block $\left[i_{1}, \ldots, i_{n-k+1}\right]$, a tree with $n-k+1$ leaves labelled by $\left\{i_{1}, \ldots, i_{n-k+1}\right\}$. There are exactly $C_{n-k+1}$ different trees (this corresponds to the choice of the grafted tree). Moreover, the tree $T_{k}$ is obtained after the first merger if the original tree is one of those, and if the chosen internal node is the leaf labelled by the block. Hence, the probability of obtaining $T_{k}$ is

$$
\frac{1}{n-1} \frac{C_{n-k+1}}{C_{n}}
$$


We note that this probability depends only of the number $k$ of leaves of the tree and not on the tree itself, hence conditionally on merging $n-k+1$ leaves, the resulting tree is still uniform among all the trees with $k$ leaves.

\section{Links with the PRUNing of Aldous's CRT}

3.1. Aldous's CRT. In [6], Aldous introduced a continuum random tree (CRT) which can be obtained as the scaling limit of critical Galton-Watson trees when the length of the branches tends to 0 . This tree can also be seen as a compact (with respect to the Gromov-Hausdorff topology) real tree. Indeed, a real tree is a metric space $(\mathcal{T}, d)$ satisfying the following two properties for every $x, y \in \mathcal{T}$ :

- (unique geodesic) There is a unique isometric map $f_{x, y}$ from $[0, d(x, y)]$ into $\mathcal{T}$ such that $f_{x, y}(0)=x$ and $f_{x, y}(d(x, y))=y$.

- (no loop) If $\varphi$ is a continuous injective map from $[0,1]$ into $\mathcal{T}$ such that $\varphi(0)=x$ and $\varphi(1)=y$, then

$$
\varphi([0,1])=f_{x, y}([0, d(x, y)]) .
$$

A rooted real tree is a real tree with a distinguished vertex denoted $\emptyset$ and called the root.

It is well known that every compact real tree can be coded by a continuous function (this coding is described below), Aldous's tree is just the real tree coded by a normalized Brownian excursion $e$. We refer to [24] for more details on real trees and their coding by continuous functions.

Remark 3.1. In fact Aldous' CRT is coded by 2e. We omit here the factor 2 for convenience but some constants may vary between this paper and Aldous' results. Our setting corresponds to the branching mechanism $\psi(\lambda)=2 \lambda^{2}$ in [15].

Let $e$ be a normalized Brownian excursion on $[0,1]$. For $s, t \in[0,1]$ we set:

$$
d(s, t)=e(s)+e(t)-2 \inf _{u \in[s \wedge t, s \vee t]} e(u) .
$$

We then define the equivalence relation $s \sim t$ iff $d(s, t)=0$ and the tree $\mathcal{T}$ is the quotient space $\mathcal{T}=[0,1] / \sim$. We denote by $p$ the canonical projection from $[0,1]$ to $\mathcal{T}$. The distance $d$ induces a distance on $\mathcal{T}$ and we keep notation $d$ for this distance. The metric space $(\mathcal{T}, d)$ is then a real tree. The metric space $(\mathcal{T}, d)$ can be seen as a rooted tree by choosing $\emptyset=p(0)$ as the root.

For $x, y \in \mathcal{T}$, we denote by $\llbracket x, y \rrbracket$ the range of the unique injective continuous path between $x$ and $y$ in $\mathcal{T}$. We also define a length measure $\ell(d x)$ on the skeleton of $\mathcal{T}$ (i.e. non-leaves vertices) by

$$
\ell(\llbracket x, y \rrbracket)=d(x, y) .
$$

Finally, for $x, y \in \mathcal{T}$, we denote by $a(x, y)$ their last common ancestor i.e. the unique point in $\mathcal{T}$ such that:

$$
\llbracket \emptyset, x \rrbracket \cap \llbracket \emptyset, y \rrbracket=\llbracket \emptyset, a(x, y) \rrbracket .
$$

For simplicity, we write for $s, t \in[0,1], a(s, t)$ for $a(p(s), p(t))$.

3.2. The interval-partition-valued process. As in $[5,1]$, we throw points on the CRT "uniformly" on the skeleton of the CRT and add more and more points as time goes by. More precisely, we consider a Poisson point measure $M(d \theta, d x)$ on $[0,+\infty) \times \mathcal{T}$ with intensity $4 d \theta \ell(d x)$. 
For $\theta>0$, we define an equivalence relation $\sim_{\theta}$ on $[0,1]$ by:

$$
s \sim_{\theta} t \Longleftrightarrow \begin{cases}s=t & \text { or } \\ M([0, \theta] \times \llbracket \emptyset, a(s, t) \rrbracket)>0 . & \end{cases}
$$

We set $\left(I_{k}^{\theta}, k \in \mathcal{K}_{\theta}\right)$ the equivalence classes associated with $\sim_{\theta}$ non-reduced to a singleton. Let us remark that each $I_{k}^{\theta}$ is an interval.

Equivalently, we define

$$
\mathcal{T}_{\theta}=\{x \in \mathcal{T}, M([0, \theta] \times \llbracket \emptyset, x \rrbracket)=0\}
$$

which is the set of vertices that have no marks on their linage. The tree $\mathcal{T}_{\theta}$ is called the pruned tree; it corresponds to the whole dust of the coalescent process. Then consider the set $\left(\mathcal{T}_{k}^{\theta}, k \in \mathcal{K}_{\theta}\right)$ of the connected components of $\mathcal{T} \backslash \mathcal{T}_{\theta}$ which are the sub-trees that are grafted on the leaves of $\mathcal{T}_{\theta}$ to get $\mathcal{T}$ (see Figure 2). Then $I_{k}^{\theta}$ is just the set of $s \in[0,1]$ such that $p(s) \in \mathcal{T}_{k}^{\theta}$.
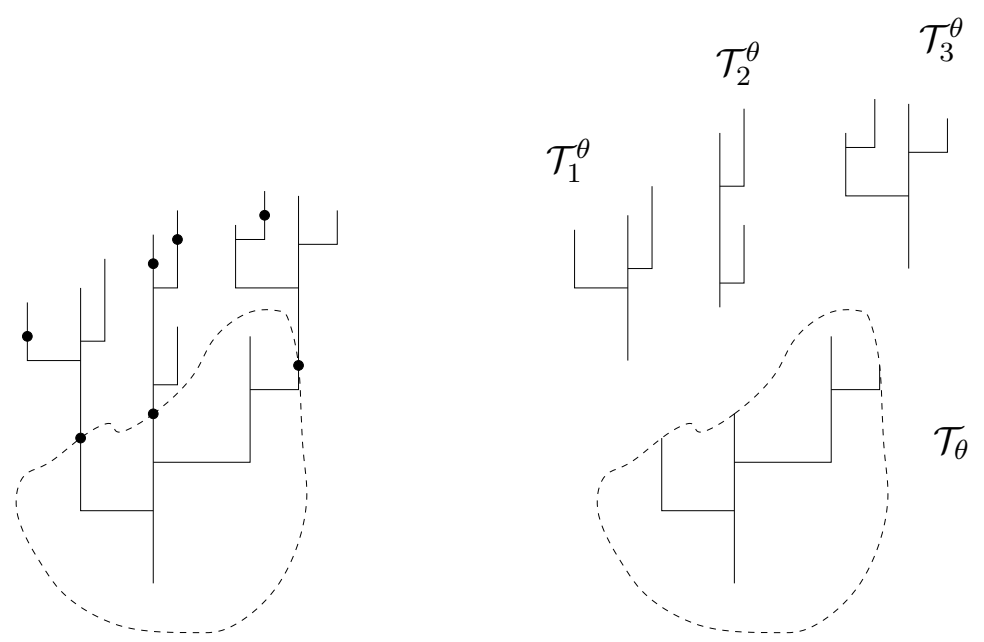

Figure 2. Left: Aldous's CRT with the marks. Right: the sub-trees constructed from the marks

By the definition of the mark process, for $\theta^{\prime}>\theta$ we have

$$
s \sim_{\theta} t \Longrightarrow s \sim_{\theta^{\prime}} t
$$

and consequently

$$
\forall k \in \mathcal{K}_{\theta}, \exists k^{\prime} \in \mathcal{K}_{\theta^{\prime}}, I_{k}^{\theta} \subset I_{k^{\prime}}^{\theta^{\prime}}
$$

Therefore, the process $\mathcal{I}=\left(\left(I_{k}^{\theta}, k \in \mathcal{K}_{\theta}\right), \theta \geq 0\right)$ can be viewed as a process where several blocks coalesce together (with part of the dust) into a single larger block. On the picture on trees, when $\theta$ increases, the number of marks also increases and when a mark appears on $\mathcal{T}_{\theta}$, some sub-trees above $\mathcal{T}_{\theta}$ coalesce with part of $\mathcal{T}_{\theta}$.

Let us remark that, as announced in the introduction, this process always has dust which corresponds to individuals that have no marks on their lineage i.e. that belong to $\mathcal{T}_{\theta}$. The dust has Lebesgue measure $\sigma_{\theta}$ :

$$
\sigma_{\theta}=\int_{0}^{1} \mathbf{1}_{\{M([0, \theta] \times \llbracket \emptyset, p(s) \rrbracket)=0\}} d s=\int_{0}^{1} \mathbf{1}_{\left\{p(s) \in \mathcal{T}_{\theta}\right\}} d s .
$$


We recall the distribution of $\left(\sigma_{\theta}, \theta \geq 0\right)$ from [8] on the size process of a tagged fragment for a self-similar fragmentation, see also [2] Proposition 9.1 or Corollary 9.2 (but with $\beta=2$ ). The distribution of $\left(\sigma_{\theta}, \theta \geq 0\right)$ under the normalized Brownian excursion measure is given by $\left(1 /\left(1+4 \tau_{\theta}\right), \theta \geq 0\right)$ where $\left(\tau_{\theta}, \theta \geq 0\right)$ is a stable subordinator with index $1 / 2$ with no drift, no killing, and Lévy measure $\left(2 \pi x^{3}\right)^{-1 / 2} d x$ on $(0, \infty)$ : for $\lambda \geq 0, \mathbb{E}\left[\mathrm{e}^{-\lambda \tau_{\theta}}\right]=\mathrm{e}^{-\theta \sqrt{2 \lambda}}$.

3.3. The reduced tree with $n$ leaves. We apply the paintbox procedure to this process. Consider $n$ independent (and independent of the process) random variables, uniformly distributed on $(0,1)$. This corresponds to choosing $n$ leaves uniformly on Aldous's CRT. Then the law of the reduced tree, $\mathcal{T}^{n}$, containing these $n$ leaves is given in [15], Section 3.3, or [7], Section 4.3. The shape of the reduced tree is a binary tree with uniform probability on all ordered binary tree with $n$ leaves. As the tree is binary, it is composed of $2 n-1$ branches with lengths $\left(h_{1}, \ldots, h_{2 n-1}\right)$ and distribution:

$$
2^{n+1} \frac{(2 n-1) !}{(n-1) !} s_{n} \mathrm{e}^{-2 s_{n}^{2}} \mathbf{1}_{\left\{h_{1}>0, \ldots, h_{2 n-1}>0\right\}} d h_{1} \cdots d h_{2 n-1},
$$

where $s_{n}=\sum_{k=1}^{2 n-1} h_{k}$ is the total length of the reduced tree.

Since the reduced tree is binary and all its edges are identically distributed, the first mark that appears on the reduced tree is uniformly distributed among all the edges and we deduce that this continuous coalescent procedure has the same structure as a $\beta(3 / 2,1 / 2)$ coalescent process. However this process is not stricto sensu a coalescent process as the time at which $n$ leaves chosen at random undergo a coalescence is not exponentially distributed, but is distributed according to an exponential random variable with (random) parameter $H_{n}$, with $H_{n}$ equal to 4 times the total length of the internal branches length. Thus $H_{n}$ is distributed as $4 \sum_{k=1}^{n-1} h_{k}$. Notice the random variables $\left(h_{1}, \ldots, h_{2 n-1}\right)$ are exchangeable and $\mathbb{E}\left[h_{1}\right]=2^{-3 / 2} \Gamma(n-1 / 2) / \Gamma(n)$. In particular, we have:

$$
\mathbb{E}\left[H_{n}\right]=4(n-1) \mathbb{E}\left[h_{1}\right]=\sqrt{2}(n-1) \frac{\Gamma\left(n-\frac{1}{2}\right)}{\Gamma(n)}=\sqrt{\frac{2}{\pi}} \lambda_{n} .
$$

So $\mathbb{E}\left[H_{n}\right]$ corresponds (up to a scaling constant) to the rate of the $\beta(3 / 2,1 / 2)$-coalescent starting with $n$ individuals.

Remark 3.2. We conjecture there exists a random time change $\left(A_{t}, t \geq 0\right)$ such that the process process $\left(\left(I_{k}^{A_{t}}, k \in \mathcal{K}_{A_{t}}\right), t \geq 0\right)$ is the interval-partition-valued process associated with the $\beta$-coalescent. However, we were not able to exhibit such a time change.

\section{Applications}

4.1. Number of coalescent events. Proposition 1.2 is just a consequence of Theorem 6.2 of [22] on the number of cuts used to isolate the root in a Galton-Watson tree with a given number of leaves. We must just remark that a binary tree with $n$ leaves has $2 n-1$ edges and that a Galton-Watson tree with binary branching conditioned to have $n$ leaves is uniformly distributed among the binary trees with $n$ leaves.

Remark 4.1. According to [1], we also have the following equality in distribution:

$$
Z \stackrel{(d)}{=} \Theta \quad \text { with } \quad \Theta=\int_{0}^{\infty} \sigma_{\theta} d \theta
$$

Notice that if a pruning mark appears twice or more on the same internal branch, only one will be taken into account as a coalescent event, and that the pruning marks which appear 
on the external branch will not be taken into account as a coalescent event. Let $X_{n}$ be the number of pruning events of the reduced tree with $n$ leaves and $X_{n}^{\prime}$ the number of coalescent events. We deduce that $X_{n}$ is stochastically larger than (or equal to) $X_{n}^{\prime}$. But the a.s. convergence which appears in [1] (see also [20] for the fluctuations) gives that a.s.:

$$
\lim _{n \rightarrow+\infty} \frac{X_{n}}{\sqrt{n}}=\sqrt{2} \Theta
$$

This implies that the number of marked external branches, say $W_{n}$, is of order $o(n)$. We shall see in Section 4.2.2 that it is in fact of order 1.

\subsection{Last coalescent event.}

4.2.1. The CRT framework. We refer to [15] for the construction of Lévy trees and their main properties. Let $\mathcal{T}$ be a continuum Lévy tree associated with the branching mechanism $\psi(\lambda)=\alpha \lambda^{2}$, i.e. coded as in Section 3.1 by a positive excursion $e$ of $\sqrt{2 \alpha} B$ where $B$ is a standard Brownian motion. We denote by $\mathbb{N}$ the "law" of this tree when the coded function $e$ is distributed according to the Itô measure (hence $\mathbb{N}$ is an infinite measure and is not really a distribution) and by $\mathbb{N}^{(r)}$ the same law when $e$ is distributed as a normalized excursion of length $r$. We denote by $\mathbf{m}$ the mass measure on the tree, i.e. the image of the Lebesgue measure on $[0,+\infty)$ by the canonical projection $p$.

Conditionally on $\mathcal{T}$, let $M$ be defined as in Section 3.2 with the intensity $2 \alpha d \theta \ell(d s)$ instead of $4 d \theta \ell(d s)$. (We introduce the parameter $\alpha$ in order to make the references to [2] easier.) Consider the pruning of the tree $\mathcal{T}$ at time $\theta>0$ :

$$
\mathcal{T}_{\theta}=\{s \in \mathcal{T} ; M([0, \theta] \times \llbracket \emptyset, s \rrbracket)=0\} .
$$

And we set $\sigma_{\theta}=\mathbf{m}\left(\mathcal{T}_{\theta}\right)$. This notation is consistent with the definition of $\sigma_{\theta}$ in Section 3.2.

Using Theorem 1.1 of [4] (see also Proposition 5.4 of [2]), we get that under $\mathbb{N}$, the pruned tree $\mathcal{T}_{\theta}$ is distributed as a Lévy tree associated with the branching mechanism $\psi_{\theta}$ defined by:

$$
\psi_{\theta}(u)=\psi(u+\theta)-\psi(\theta) .
$$

Moreover, using Lemma 3.8 of [2], we have the following Girsanov formula that links the law of $\mathcal{T}_{\theta}$ with that of $\mathcal{T}$ : for every nonnegative measurable functional $F$ on the space of trees, we have:

$$
\mathbb{N}\left[F\left(\mathcal{T}_{\theta}\right)\right]=\mathbb{N}\left[F(\mathcal{T}) \mathrm{e}^{-\alpha \theta^{2} \sigma}\right] .
$$

Let $n$ be a positive integer. We consider under $\mathbb{N}\left(\right.$ or $\left.\mathbb{N}^{(r)}\right)$ conditionally given the tree $\mathcal{T}, n$ leaves $x_{1}, \ldots, x_{n}$ i.i.d., uniformly chosen on the set of leaves, i.e. sampled with the probability $\mathbf{m}(d x) / \sigma$. For $\theta>0$, let $\left(\mathcal{T}^{j}, j \in J\right)$ be the connected components of $\mathcal{T} \backslash \mathcal{T}_{\theta}$. We write $Y_{\theta}^{0}(n)=\sum_{\ell=1}^{n} \mathbf{1}_{\left\{x_{\ell} \in \mathcal{T}_{\theta}\right\}}$ the number of chosen leaves on the subtree $\mathcal{T}_{\theta}$, and for $k \geq 1$, $Y_{\theta}^{k}(n)=$ Card $\left\{j \in J ; \sum_{\ell=1}^{n} \mathbf{1}_{\left\{x_{\ell} \in \mathcal{T}^{j}\right\}}=k\right\}$ the number of subtrees with exactly $k$ chosen leaves. In particular, we have:

$$
Y_{\theta}^{0}(n)+\sum_{k \geq 1} k Y_{\theta}^{k}(n)=n .
$$

We set $N_{\theta}(n)=\sum_{k \geq 0} Y_{\theta}^{k}(n)$ the number of chosen leaves on $\mathcal{T}_{\theta}$ plus the number of subtrees with chosen leaves. For convenience, we shall consider:

$$
Y_{\theta}(n)=\sum_{k \geq 2} Y_{\theta}^{k}(n)=N_{\theta}(n)-Y_{\theta}^{0}(n)-Y_{\theta}^{1}(n) .
$$


Let $\mathcal{T}^{n}$ be the reduced tree of the chosen leaves: that is the smallest connected component of $\mathcal{T}$ containing the root $\emptyset$ and $\left\{x_{i}, 1 \leq i \leq n\right\}$. Let $\mathcal{T}_{\theta}^{n}$ be the reduced tree $\mathcal{T}^{n}$ pruned at time $\theta>0$ :

$$
\mathcal{T}_{\theta}^{n}=\left\{s \in \mathcal{T}^{n} ; M([0, \theta] \times \llbracket 0, s \rrbracket)=0\right\} .
$$

Notice that $N_{\theta}$ is the number of leaves of $\mathcal{T}_{\theta}^{n}$ (with the convention that the root is not a leaf). Define the last pruning event as:

$$
L_{n}=\inf \left\{\theta>0 ; N_{\theta}(n)=1\right\} .
$$

We define:

$$
U_{n}=N_{L_{n}-}(n), \quad V_{n}=Y_{L_{n}-}^{0}(n)+Y_{L_{n}-}^{1}(n), \quad \text { and } \quad W_{n}=Y_{L_{n}-}^{1}(n) .
$$

We can interpret $U_{n}$ as the number of leaves of the pruned reduced tree, $V_{n}-W_{n}$ as the number of chosen leaves of the pruned reduced tree and $W_{n}$ as the number of subtrees with only one chosen leaf just before the last pruning event.

4.2.2. Proof of Proposition 1.3. Let $B_{n}$ be the number of blocks and $E_{n}$ be the number of singletons involved in the last coalescent event of $\left(\Pi^{[n]}(t), t \geq 0\right)$. Using the link with the pruning of CRT from the previous Section, we have that $\left(B_{n}, E_{n}\right)$ is distributed as $\left(U_{n}, V_{n}\right)$ under $\mathbb{N}^{(1)}$.

Following Remark 4.1, we can see $V_{n}$ as the sum of $V_{n}-W_{n}$ (number of leaves of $\mathcal{T}^{n}$ with no mark before the last pruning event) and the number $W_{n}$ of leaves of $\mathcal{T}^{n}$ with no mark on their ancestral lineage until the mark corresponding to the last coalescent pruning but for the external branch, where there is at least one mark.

Before giving the asymptotic distribution of $\left(U_{n}, V_{n}\right)$, we need to introduce some notations.

For $a \geq 0, b \geq 0$ such that $a+b>0$ we define $\Delta_{0}(a, b)$ as:

$$
\Delta_{0}(a, b)= \begin{cases}\frac{1}{\sqrt{|1+b-2 a|}} \log \left(\frac{1+\sqrt{b}+\sqrt{|1+b-2 a|}}{1+\sqrt{b}-\sqrt{|1+b-2 a|}}\right) & \text { if } 1+b-2 a \neq 0, \\ \frac{2}{1+\sqrt{b}} & \text { if } \quad 1+b-2 a=0 .\end{cases}
$$

It is easy to check that the function $\Delta_{0}$ is continuous in $(a, b)$ and that:

$$
\lim _{(a, b) \rightarrow(0,0)} \Delta_{0}(a, b)+\log (\sqrt{b}+a)=\log (2) .
$$

We set for $a \geq 0$ and $b \geq 0$ (with the convention $I(0,0)=1$ ):

$$
I(a, b)=1+\log (2)-\log (\sqrt{b}+a)-\Delta_{0}(a, b) .
$$

Notice that the function $I$ is continuous on $[0,+\infty)^{2}$ (and in particular at $\left.(0,0)\right)$.

We shall prove the next result in Section 5 .

Proposition 4.2. Under $\mathbb{N}^{(1)}$, as $n$ goes to infinity, $\left(U_{n}, V_{n}, V_{n}\right)$ converges, in distribution, to a finite random variable $(U, V, W)$. And the distribution of $(U, V, W)$ is characterized by the following generating function, for $\rho, \rho_{0}, \rho_{1} \in[0,1]$ :

$$
\Psi\left(\rho, \rho_{0}, \rho_{1}\right)=\mathbb{E}\left[\rho^{U-V} \rho_{0}^{V-W} \rho_{1}^{W}\right]=\rho I\left(1-\frac{\rho+\rho_{1}}{2}, 1-\rho_{0}\right) .
$$

Notice that the random variable $U-V-1$ is distributed as $W$. Since $W \leq V$, this implies that $U-V-1$ is stochastically smaller than $V$. This last remark and Proposition 4.2 readily implies Proposition 1.3. 
The generating function of $V-W$ is given, for $\rho_{0} \in[0,1]$ by $\mathbb{E}\left[\rho_{0}^{V-W}\right]=I\left(0,1-\rho_{0}\right)$, that is:

$$
\mathbb{E}\left[\rho_{0}^{V-W}\right]=1+\log (2)-\log \left(\sqrt{1-\rho_{0}}\right)-\frac{1}{\sqrt{2-\rho_{0}}} \log \left(\frac{1+\sqrt{1-\rho_{0}}+\sqrt{2-\rho_{0}}}{1+\sqrt{1-\rho_{0}}-\sqrt{2-\rho_{0}}}\right) .
$$

The generating function of $W$ is given by, for $\rho_{1} \in[0,1]$ by:

$$
\mathbb{E}\left[\rho_{1}^{W}\right]=I\left(\frac{1-\rho_{1}}{2}, 0\right)=1+\log (4)-\log \left(1-\rho_{1}\right)-\frac{1}{\sqrt{\rho_{1}}} \log \left(\frac{1+\sqrt{\rho_{1}}}{1-\sqrt{\rho_{1}}}\right) .
$$

\section{Proof of Proposition 4.2}

In order to compute the generating function that appears in Proposition 4.2, it is easier to work first under $\mathbb{N}$ (and then condition on $\sigma$ to have the result under $\mathbb{N}^{(1)}$ and to consider a Poissonian number of chosen leaves. Let $\lambda>0$. Under $\mathbb{N}$ or $\mathbb{N}^{(r)}$, conditionally given the tree $\mathcal{T}$, we consider a Poisson point measure $\mathcal{N}=\sum_{i \in I} \delta_{x_{i}}$ on $\mathcal{T}$ with intensity $\lambda \mathbf{m}$. We denote by $\tilde{N}=\mathcal{N}(\mathcal{T})$ the number of chosen leaves. The law of the total mass $\sigma=\mathbf{m}(\mathcal{T})$ of $\mathcal{T}$ under $\mathbb{N}$ is given by the following Laplace transform:

$$
\mathbb{N}\left[1-\mathrm{e}^{-\kappa \sigma}\right]=\psi^{-1}(\kappa)=\sqrt{\kappa / \alpha}
$$

Conditionally on $\sigma$, the random variable $\tilde{N}$ is Poisson with parameter $\lambda \sigma$. Therefore, by first conditioning on $\sigma$, we get for $k \geq 1$ :

$$
\mathbb{N}[\tilde{N}=k]=\frac{\lambda^{k}}{k !} \mathbb{N}\left[\sigma^{k} \mathrm{e}^{-\lambda \sigma}\right]=\frac{1}{2 \sqrt{\pi}} \sqrt{\frac{\lambda}{\alpha}} \frac{\Gamma\left(k-\frac{1}{2}\right)}{\Gamma(k+1)} .
$$

Let $\theta>0$. From the special Markov property, Theorem 5.6 in [2] (see also [3]), we get that under $\mathbb{N}$, conditionally on $\sigma_{\theta}$, the random variables $\left(Y_{\theta}^{k}(\tilde{N}), k \geq 0\right)$ are independent, $Y_{\theta}^{0}(\tilde{N})$ is Poisson with parameter $\lambda \sigma_{\theta}$ and for $k \geq 1, Y_{\theta}^{k}(\tilde{N})$ is Poisson with parameter $2 \alpha \theta \sigma_{\theta} \mathbb{N}[\tilde{N}=k]$.

For $a \in[0,1]$, we set:

$$
f_{\theta}(a)=\mathbb{N}\left[a^{\tilde{N}} \rho^{Y_{\theta}(\tilde{N})} \rho_{0}^{Y_{\theta}^{0}(\tilde{N})} \rho_{1}^{Y_{\theta}^{1}(\tilde{N})} \mathbf{1}_{\{\tilde{N}>0\}}\right]
$$

Lemma 5.1. We have:

$$
f_{\theta}(a)=\theta+\sqrt{\frac{\lambda}{\alpha}}-\sqrt{\delta_{0}+2 \delta_{1} \sqrt{1-a}-\delta_{2} a}
$$

with

$$
\delta_{0}=\theta^{2}+\frac{\lambda}{\alpha}+2 \theta \sqrt{\frac{\lambda}{\alpha}}(1-\rho), \quad \delta_{1}=\theta \rho \sqrt{\frac{\lambda}{\alpha}}, \quad \delta_{2}=\frac{\lambda}{\alpha} \rho_{0}-\theta \sqrt{\frac{\lambda}{\alpha}}\left(\rho-\rho_{1}\right) .
$$

Notice that:

$$
\delta_{0}-\delta_{2}=\theta^{2}+\frac{\lambda}{\alpha}\left(1-\rho_{0}\right)+\theta \sqrt{\frac{\lambda}{\alpha}}\left(2-\rho-\rho_{1}\right) \geq \theta^{2}>0 .
$$

Consequently the right hand side in (16) is well defined. 
Proof. We set $\mu=-\log (\rho)$ and $\mu_{i}=-\log \left(\rho_{i}\right)$ for $i \in\{0,1\}$ and $\kappa=-\log (a)$. We have:

$$
\begin{aligned}
& f_{\theta}(a) \\
& \quad= \mathbb{N}\left[\mathrm{e}^{-\mu_{0} Y_{\theta}^{0}(\tilde{N})-\mu_{1} Y_{\theta}^{1}(\tilde{N})-\mu Y_{\theta}(\tilde{N})-\kappa \tilde{N}}-\mathbf{1}_{\{\tilde{N}=0\}}\right] \\
& \quad=\mathbb{N}\left[\mathbb{N}\left[\mathrm{e}^{-\left(\mu_{0}+\kappa\right) Y_{\theta}^{0}(\tilde{N})} \mid \sigma_{\theta}\right] \tilde{\mathbb{N}}\left[\mathrm{e}^{-\left(\mu_{1}+\kappa\right) Y_{\theta}^{1}(\tilde{N})} \mid \sigma_{\theta}\right] \prod_{k \geq 2} \mathbb{N}\left[\mathrm{e}^{-(\mu+k \kappa) Y_{\theta}^{k}(\tilde{N})} \mid \sigma_{\theta}\right]\right]-\mathbb{N}[\tilde{N}=0],
\end{aligned}
$$

using Equation (8) and the independence of the variables $Y_{\theta}^{k}(\tilde{N})$ conditionally given $\sigma_{\theta}$. Now, as the variables $Y_{\theta}^{k}(\tilde{N})$ are conditionally given $\sigma_{\theta}$ Poisson variables, and thanks to (15), we have:

with

$$
f_{\theta}(a)=\mathbb{N}\left[\mathrm{e}^{-\gamma \sigma_{\theta}}-\mathrm{e}^{-\lambda \sigma}\right]
$$

$$
\begin{aligned}
\gamma= & \lambda\left(1-\mathrm{e}^{-\left(\kappa+\mu_{0}\right)}\right)+2 \alpha \theta \lambda \mathbb{N}\left[\sigma \mathrm{e}^{-\lambda \sigma}\right]\left(1-\mathrm{e}^{-\left(\kappa+\mu_{1}\right)}\right)+2 \alpha \theta \sum_{k \geq 2} \frac{\lambda^{k}}{k !} \mathbb{N}\left[\sigma^{k} \mathrm{e}^{-\lambda \sigma}\right]\left(1-\mathrm{e}^{-(k \kappa+\mu)}\right) \\
= & \lambda\left(1-\mathrm{e}^{-\left(\kappa+\mu_{0}\right)}\right)+2 \alpha \theta \lambda \mathbb{N}\left[\sigma \mathrm{e}^{-\lambda \sigma}\right]\left(1-\mathrm{e}^{-\left(\kappa+\mu_{1}\right)}\right) \\
& +2 \alpha \theta\left(\mathbb{N}\left[1-\mathrm{e}^{-\lambda \sigma}\right]\left(1-\mathrm{e}^{-\mu}\right)-\lambda \mathbb{N}\left[\sigma \mathrm{e}^{-\lambda \sigma}\right]\left(1-\mathrm{e}^{-(\mu+\kappa)}\right)+\mathrm{e}^{-\mu} \mathbb{N}\left[1-\mathrm{e}^{-\lambda\left(1-\mathrm{e}^{-\kappa}\right) \sigma}\right]\right) .
\end{aligned}
$$

We now use Formula (14) to get

$$
\begin{aligned}
\gamma & =\lambda\left(1-\mathrm{e}^{-\left(\kappa+\mu_{0}\right)}\right)+\theta \sqrt{\lambda \alpha} \mathrm{e}^{-\kappa}\left(\mathrm{e}^{-\mu}-\mathrm{e}^{-\mu_{1}}\right)+2 \theta \sqrt{\lambda \alpha}\left(1-\mathrm{e}^{-\mu}\right)+2 \theta \mathrm{e}^{-\mu} \sqrt{\lambda \alpha} \sqrt{1-\mathrm{e}^{-\kappa}} \\
& =2 \theta \sqrt{\lambda \alpha}\left(1-\mathrm{e}^{-\mu}\right)+\lambda+2 \theta \mathrm{e}^{-\mu} \sqrt{\lambda \alpha} \sqrt{1-a}-a\left(\lambda \mathrm{e}^{-\mu_{0}}-\theta \sqrt{\lambda \alpha}\left(\mathrm{e}^{-\mu}-\mathrm{e}^{-\mu_{1}}\right)\right) .
\end{aligned}
$$

Using the special Markov property of [2], Theorem 5.6, we get that, conditionally given $\sigma_{\theta}, \sigma$ is distributed as $\sigma_{\theta}+\sum \sigma_{i}$ where the $\sigma_{i}$ 's are the atoms of a Poisson point measure of intensity $2 \alpha \theta \sigma_{\theta} \mathbb{N}[d \sigma]$. This yields:

$$
f_{\theta}(a)=\mathbb{N}\left[\mathrm{e}^{-\gamma \sigma_{\theta}}-\mathrm{e}^{-\sigma_{\theta}(\lambda+2 \theta \sqrt{\lambda \alpha})}\right] .
$$

To conclude, we use Girsanov Formula (7) to get:

$$
\begin{aligned}
f_{\theta}(a) & =\mathbb{N}\left[\mathrm{e}^{-\left(\gamma+\alpha \theta^{2}\right) \sigma}-\mathrm{e}^{-\sigma\left(\alpha \theta^{2}+\lambda+2 \theta \sqrt{\lambda \alpha}\right)}\right] \\
& =\theta+\sqrt{\frac{\lambda}{\alpha}}-\sqrt{\theta^{2}+\frac{\gamma}{\alpha}} .
\end{aligned}
$$

This gives the result.

Let us set

$$
A_{n}=\mathbb{N}^{(1)}\left[\mathrm{e}^{-\mu\left(U_{n}-V_{n}\right)-\mu_{0}\left(V_{n}-W_{n}\right)-\mu_{1} W_{n}}\right] .
$$

To prove Proposition 4.2, it is enough to prove that:

$$
\lim _{n \rightarrow+\infty} A_{n}=\rho I\left(1-\frac{\rho+\rho_{1}}{2}, 1-\rho_{0}\right) .
$$

As the CRT is coded by a Brownian excursion, it enjoys a scaling property, namely the law of $r \mathcal{T}$ under $\mathbb{N}^{\left(r^{2}\right)}$ is those of $\mathcal{T}$ under $\mathbb{N}^{(1)}$ (where $r \mathcal{T}$ means that we multiply the distance on the tree by a factor $r$ ). Consequently, the mark process (defined as a Poisson point measure 
with intensity proportional to the length measure) also satisfies a scaling property. It is then easy to deduce that the law of $\left(U_{n}, V_{n}, W_{n}\right)$ doesn't depend on $\sigma$. So, we have:

$$
A_{n}=\mathbb{N}^{(r)}\left[\mathrm{e}^{-\mu\left(U_{n}-V_{n}\right)-\mu_{0}\left(V_{n}-W_{n}\right)-\mu_{1} W_{n}}\right]
$$

for every positive $r$. We set:

$$
\mathcal{A}_{n}=A_{n} \mathbb{N}\left[\sigma^{n} \mathrm{e}^{-\lambda \sigma}\right]
$$

By conditioning on $\sigma$, we get:

$$
\mathcal{A}_{n}=\mathbb{N}\left[\sigma^{n} \mathrm{e}^{-\lambda \sigma} \mathrm{e}^{-\mu\left(U_{n}-V_{n}\right)-\mu_{0}\left(V_{n}-W_{n}\right)-\mu_{1} W_{n}}\right] .
$$

For $n \geq 1$, we set:

$$
F_{\theta}(n, r)=\mathbb{N}^{(r)}\left[\rho^{Y_{\theta}(n)} \rho_{0}^{Y_{\theta}^{0}(n)} \rho_{1}^{Y_{\theta}^{1}(n)}\right] \quad \text { and } \quad H_{n}(\theta)=\mathbb{N}\left[F_{\theta}(n, \sigma) \sigma^{n} \mathrm{e}^{-\lambda \sigma}\right] .
$$

Recall:

$$
\mathbb{N}\left[\sigma^{n} \mathrm{e}^{-\lambda \sigma}\right]=\lambda^{-n} n ! \mathbb{N}[\tilde{N}=n]=\frac{1}{2 \sqrt{\alpha \pi} \lambda^{n-\frac{1}{2}}} \Gamma\left(n-\frac{1}{2}\right) .
$$

Therefore, we have:

$$
f_{\theta}(a)=\mathbb{N}\left[a^{\tilde{N}} F_{\theta}(\tilde{N}, \sigma) \mathbf{1}_{\{\tilde{N}>0\}}\right],
$$

and, thanks to (21), for $n \geq 1$ :

$$
f_{\theta}^{(n)}(0)=n ! \mathbb{N}\left[F_{\theta}(n, \sigma) \mathbf{1}_{\{\tilde{N}=n\}}\right]=\lambda^{n} H_{n}(\theta) .
$$

We use the description of [15] of the reduced tree spanned by $n$ leaves under the $\sigma$-finite measure $\mathbb{N}$ : it is a uniform binary tree with $n$ leaves and with edge lengths i.i.d. and "distributed" as $\alpha d h$. We denote by $\llbracket \emptyset, x_{1} \rrbracket$ the edge of the reduced tree attached to the root, and by $H$ its length. The time $L_{n}$ at which the last coalescent event occurs is just the first time $\theta$ at which a mark appears on $\llbracket \emptyset, x_{1} \rrbracket$ and therefore it is, conditionally given $H=h$, exponentially distributed with parameter $2 \alpha h$. Moreover, if we denote by $\mathcal{T}^{(1)}$ and $\mathcal{T}^{(2)}$ the two sub-trees attached to $x_{1}$, and $\sigma^{(1)}, \sigma^{(2)}$ respectively their total mass, we have:

$$
\sigma=\sigma^{(1)}+\sigma^{(2)}+\sum_{i \in I^{\prime}} \sigma_{i}
$$

where the $\sigma_{i}$ 's are the total mass of the sub-trees attached on the edge $\llbracket \emptyset, x_{1} \rrbracket$. The random measure $\sum_{i \in I^{\prime}} \sigma_{i}$ is independent of $\sigma^{(1)}, \sigma^{(2)}$ and is, conditionally on $\{H=h\}$, distributed as a Poisson point measure with intensity $2 \alpha h \mathbb{N}[d \sigma]$. Eventually, the two reduced sub-trees attached to $x_{1}$ are independent and distributed as uniform binary trees with respectively $k$ and $n-k$ leaves $(0<k<n)$. Recall that $2 \alpha h \mathbb{N}\left[1-\mathrm{e}^{-\lambda \sigma}\right]=2 \sqrt{\alpha \lambda} h$. We deduce from this description that:

$$
\begin{aligned}
\mathcal{A}_{n} & =\int_{0}^{\infty} \alpha \mathrm{e}^{-2 \sqrt{\alpha \lambda} h} d h \int_{0}^{\infty} 2 \alpha h \mathrm{e}^{-2 \alpha h \theta} d \theta \sum_{k=1}^{n-1}\left(\begin{array}{l}
n \\
k
\end{array}\right) H_{k}(\theta) H_{n-k}(\theta) \\
& =\lambda^{-n} \int_{0}^{\infty} \frac{d \theta}{(\theta+\sqrt{\lambda / \alpha})^{2}} G_{n}(\theta),
\end{aligned}
$$


with

$$
G_{n}(\theta)=\frac{\lambda^{n}}{2} \sum_{k=1}^{n-1}\left(\begin{array}{l}
n \\
k
\end{array}\right) H_{k}(\theta) H_{n-k}(\theta)=\frac{1}{2} \sum_{k=1}^{n-1}\left(\begin{array}{l}
n \\
k
\end{array}\right) f_{\theta}^{(k)}(0) f_{\theta}^{(n-k)}(0) .
$$

Let $c_{0}=\theta+\sqrt{\lambda / \alpha}$ and $g=c_{0}-f_{\theta}$, so that:

$$
g(a)=\sqrt{\delta_{0}+2 \delta_{1} \sqrt{1-a}-\delta_{2} a}
$$

If $h$ is a function, we denote $\partial_{a=0}^{n} h(a)$ for $h^{(n)}(0)$. Then, using the formula for the $n$-th derivative of a product of functions, we have for $n \geq 2$ :

$$
G_{n}(\theta)=\frac{1}{2} \sum_{k=1}^{n-1}\left(\begin{array}{l}
n \\
k
\end{array}\right) g^{(k)}(0) g^{(n-k)}(0)=\frac{1}{2} \partial_{a=0}^{n} g^{2}(a)-g(0) g^{(n)}(0) .
$$

That is, since $\partial_{a=0}^{n} \sqrt{1-a}=-\Gamma\left(n-\frac{1}{2}\right) / 2 \sqrt{\pi}$ :

$$
G_{n}(\theta)=\delta_{1} \partial_{a=0}^{n} \sqrt{1-a}-\sqrt{\delta_{0}+2 \delta_{1}} g^{(n)}(0)=-\delta_{1} \frac{\Gamma\left(n-\frac{1}{2}\right)}{2 \sqrt{\pi}}-\sqrt{\delta_{0}+2 \delta_{1}} g^{(n)}(0) .
$$

The next Lemma gives an equivalent expression for $g^{(n)}(0)$.

Lemma 5.2. We have:

$$
\lim _{n \rightarrow+\infty}-\frac{g^{(n)}(0)}{\Gamma\left(n-\frac{1}{2}\right)}=\frac{\delta_{1}}{2 \sqrt{\pi}} \frac{1}{\sqrt{\delta_{0}-\delta_{2}}} .
$$

Proof. Using that the density, which corresponds to the density of the 1/2-stable subordinator with no drift:

has Laplace transform:

$$
h(x)=\frac{\delta_{1} r}{\sqrt{\pi}} \frac{1}{x^{3 / 2}} \mathrm{e}^{-\delta_{1}^{2} r^{2} / x} \mathbf{1}_{\{x>0\}}
$$

we can write:

$$
\int_{0}^{+\infty} \mathrm{e}^{-\lambda x} h(x) d x=\mathrm{e}^{-2 \delta_{1} r \sqrt{\lambda}}
$$

$$
\begin{aligned}
g(a) & =\frac{1}{2 \sqrt{\pi}} \int_{0}^{\infty} \frac{d r}{r^{3 / 2}}\left(1-\mathrm{e}^{-\left(\delta_{0}+2 \delta_{1} \sqrt{1-a}-\delta_{2} a\right) r}\right) \\
& =\frac{1}{2 \pi} \int_{0}^{\infty} \frac{d r}{r^{3 / 2}} \int_{0}^{\infty} \delta_{1} r \frac{d x}{x^{3 / 2}} \mathrm{e}^{-\delta_{1}^{2} r^{2} / x}\left(1-\mathrm{e}^{-\delta_{0} r-x+a\left(\delta_{2} r+x\right)}\right) .
\end{aligned}
$$

We deduce, with $y=r / x$ that for $n \geq 1$ :

$$
\begin{aligned}
-g^{(n)}(0) & =\frac{1}{2 \pi} \int_{0}^{\infty} \frac{d r}{r^{3 / 2}} \int_{0}^{\infty} \delta_{1} r \frac{d x}{x^{3 / 2}}\left(\delta_{2} r+x\right)^{n} \mathrm{e}^{-\delta_{0} r-x-\delta_{1}^{2} r^{2} / x} \\
& =\frac{\delta_{1}}{2 \pi} \int_{0}^{\infty} r^{n-1} d r \int_{0}^{\infty} \frac{d y}{\sqrt{y}}\left(\frac{1+\delta_{2} y}{y}\right)^{n} \mathrm{e}^{-r\left(\delta_{0}+\frac{1}{y}+\delta_{1}^{2} y\right)} \\
& =\frac{\delta_{1} \Gamma(n)}{2 \pi} \int_{0}^{\infty} \frac{d y}{\sqrt{y}}\left(\frac{1+\delta_{2} y}{y}\right)^{n} \frac{1}{\left(\delta_{0}+\frac{1}{y}+\delta_{1}^{2} y\right)^{n}} \\
& =\frac{\delta_{1} \Gamma(n)}{2 \pi} \int_{0}^{\infty} \frac{d y}{\sqrt{y}} \varphi(y)^{n}
\end{aligned}
$$


with

$$
\varphi(y)=\frac{1+\delta_{2} y}{1+\delta_{0} y+\delta_{1}^{2} y^{2}}
$$

To get an equivalent expression for $g^{(n)}(0)$, we use Laplace's method. Notice that $\varphi(0)=1$ and $\varphi^{\prime}(0)=\delta_{2}-\delta_{0}<0$ thanks to $(17)$. Notice that $\delta_{0}>0$ and $\delta_{1}>0$.

The only root to $\varphi(y)=1$ is 0 . Let us assume there is a root to $\varphi(y)=-1$. This implies that $1+\delta_{0} y+\delta_{1}^{2} y^{2}+1+\delta_{2} y=0$ that is:

$$
\left(\delta_{0}+\delta_{2}\right) y+\delta_{1}^{2} y^{2}=-2 .
$$

But we have:

$$
\delta_{0}+\delta_{2} \geq \theta^{2}+\frac{\lambda}{\alpha}-\theta \sqrt{\lambda} \alpha>0 .
$$

Therefore, $|\varphi(y)| \in(-1,1)$ for $y>0$. Since, $\lim _{y \rightarrow+\infty} \varphi(y)=0$.

The discussion above proves that:

$$
\exists \delta_{0}>0, \forall \delta \in\left(0, \delta_{0}\right), \forall x \geq \delta,|\varphi(x)| \leq \varphi(\delta)<1 .
$$

Moreover, we have:

$$
\lim _{x \downarrow 0} \frac{\log (\varphi(x))}{\left(\delta_{2}-\delta_{0}\right) x}=1 .
$$

Therefore we can apply the standard Laplace's method and get the following asymptotic in $n$ :

$$
-g^{(n)}(0) \sim \frac{\delta_{1} \Gamma(n)}{2 \pi} \int_{0}^{\infty} \frac{d y}{\sqrt{y}} \mathrm{e}^{-n\left(\delta_{0}-\delta_{2}\right) y}=\frac{\delta_{1} \Gamma(n)}{2 \pi} \frac{\Gamma(1 / 2)}{\sqrt{n\left(\delta_{0}-\delta_{2}\right)}} \sim \frac{\delta_{1} \Gamma\left(n-\frac{1}{2}\right)}{2 \sqrt{\pi}} \frac{1}{\sqrt{\delta_{0}-\delta_{2}}},
$$

where $a_{n} \sim b_{n}$ means $\lim _{n \rightarrow+\infty} a_{n} / b_{n}=1$.

Recall the notations of Proposition 4.2:

$$
\Psi\left(\rho, \rho_{0}, \rho_{1}\right)=\rho I\left(1-\frac{\rho+\rho_{1}}{2}, 1-\rho_{0}\right) .
$$

Lemma 5.3. We have, for $\rho, \rho_{0}, \rho_{1} \in[0,1]$ :

$$
\lim _{n \rightarrow+\infty} \mathbb{N}^{(1)}\left[\rho^{U_{n}-V_{n}} \rho_{0}^{V_{n}-W_{n}} \rho_{1}^{W_{n}}\right]=\Psi\left(\rho, \rho_{0}, \rho_{1}\right) .
$$

Notice that $\Psi(1,1,1)=I(0,0)=1$. This implies that $\left(U_{n}, V_{n}, W_{n}\right)$ converge in distribution, as $n$ goes to infinity, to an a.s. finite random variable $(U, V, W)$ and that the generating function of $(U-V, V-W, W)$ is given by $\Psi$.

Proof. On the one hand, we deduce from (24) and Lemma 5.2 that:

$$
\lim _{n \rightarrow+\infty} \frac{G_{n}(\theta)}{\Gamma\left(n-\frac{1}{2}\right)}=-\frac{\delta_{1}}{2 \sqrt{\pi}}+\sqrt{\delta_{0}+2 \delta_{1}} \frac{\delta_{1}}{2 \sqrt{\pi}} \frac{1}{\sqrt{\delta_{0}-\delta_{2}}}=\frac{\delta_{1}}{2 \sqrt{\pi}}\left(\frac{\sqrt{\delta_{0}+2 \delta_{1}}}{\sqrt{\delta_{0}-\delta_{2}}}-1\right) .
$$

On the other hand, we deduce from (20) (by considering $\rho=\rho_{0}=\rho_{1}=1$ ) and (21) that:

$$
H_{n}(\theta) \leq \mathbb{N}\left[\sigma^{n} \mathrm{e}^{-\lambda \sigma}\right]=\frac{1}{2 \sqrt{\alpha \pi} \lambda^{n-\frac{1}{2}}} \Gamma\left(n-\frac{1}{2}\right) .
$$

Recall (6). By decomposing an ordered binary tree with $n$ labelled leaves in two ordered binary sub-trees attached to closest node of the root, we get:

$$
\sum_{k=1}^{n-1}\left(\begin{array}{l}
n \\
k
\end{array}\right) C_{k} C_{n-k}=C_{n} .
$$


This readily implies:

$$
\sum_{k=1}^{n-1}\left(\begin{array}{l}
n \\
k
\end{array}\right) \Gamma\left(k-\frac{1}{2}\right) \Gamma\left(n-k-\frac{1}{2}\right)=4 \sqrt{\pi} \Gamma\left(n-\frac{1}{2}\right) .
$$

We deduce from the first equality of (23) and (25) that:

$$
G_{n}(\theta)=\frac{\lambda^{n}}{2} \sum_{k=1}^{n-1}\left(\begin{array}{l}
n \\
k
\end{array}\right) H_{k}(\theta) H_{n-k}(\theta) \leq \frac{\lambda}{2 \alpha \sqrt{\pi}} \Gamma\left(n-\frac{1}{2}\right) .
$$

This implies that:

$$
\frac{G_{n}(\theta)}{\Gamma\left(n-\frac{1}{2}\right)} \leq \frac{\lambda}{2 \alpha \sqrt{\pi}}
$$

By dominated convergence, we deduce from (22) that:

$$
\begin{aligned}
\lim _{n \rightarrow+\infty} \frac{\lambda^{n} \mathcal{A}_{n}}{\Gamma\left(n-\frac{1}{2}\right)} & =\int_{0}^{\infty} \frac{d \theta}{(\theta+\sqrt{\lambda / \alpha})^{2}} \lim _{n \rightarrow+\infty} \frac{G_{n}(\theta)}{\Gamma\left(n-\frac{1}{2}\right)} \\
& =\frac{\sqrt{\lambda}}{2 \sqrt{\alpha \pi}} \rho \int_{0}^{\infty} \frac{\theta d \theta}{(\theta+\sqrt{\lambda / \alpha})^{2}}\left(\frac{\theta+\sqrt{\frac{\lambda}{\alpha}}}{\sqrt{\theta^{2}+\theta \sqrt{\frac{\lambda}{\alpha}}\left(2-\rho-\rho_{1}\right)+\frac{\lambda}{\alpha}\left(1-\rho_{0}\right)}}\right) \\
& =\frac{\sqrt{\lambda}}{2 \sqrt{\alpha \pi}} \rho \int_{0}^{\infty} \frac{\theta d \theta}{(\theta+1)^{2}}\left(\frac{\theta+1}{\sqrt{\theta^{2}+\theta\left(2-\rho-\rho_{1}\right)+\left(1-\rho_{0}\right)}}-1\right) .
\end{aligned}
$$

We deduce from (19) that:

$$
\begin{aligned}
\lim _{n \rightarrow+\infty} A_{n} & =\frac{2 \sqrt{\alpha \pi}}{\sqrt{\lambda}} \lim _{n \rightarrow+\infty} \frac{\lambda^{n} \mathcal{A}_{n}}{\Gamma\left(n-\frac{1}{2}\right)} \\
& =\rho \int_{0}^{\infty} \frac{\theta d \theta}{(\theta+1)^{2}}\left(\frac{\theta+1}{\sqrt{\theta^{2}+\theta\left(2-\rho-\rho_{1}\right)+\left(1-\rho_{0}\right)}}-1\right) .
\end{aligned}
$$

Then, we use Lemma 5.5 below to conclude.

Before stating and proving Lemma 5.5, we first give a preliminary result.

Lemma 5.4. For $a \geq 0, b \geq 0$ and $a+b>0$, we have $\Delta(a, b)=\Delta_{0}(a, b)$.

Proof. We first assume $1+b-2 a>0$. From (27), we have:

$$
\begin{aligned}
-\partial_{a} \Delta(a, b) & =\int_{0}^{\infty} \frac{\theta d \theta}{(\theta+1)\left(\theta^{2}+2 a \theta+b\right)^{3 / 2}} \\
& =-2 \partial_{b} I(a, b) \\
& =-\frac{\Delta(a, b)}{1+b-2 a}+\frac{1}{1+b-2 a} \frac{\sqrt{b}+1}{\sqrt{b}+a} .
\end{aligned}
$$

Then, by computing the derivative $\partial_{a} \Delta_{0}$, we deduce that $\Delta(a, b)=\Delta_{0}(a, b)+h_{b}(a)$, for a function $h_{b}$ solving:

$$
-h_{b}^{\prime}(a)=-\frac{h_{b}(a)}{1+b-2 a}
$$


that is, for some function $c_{+}$:

$$
h_{b}(a)=\frac{c_{+}(b)}{\sqrt{1+b-2 a}} .
$$

Similarly, we have that for $1+b-2 a<0$,

$$
\Delta(a, b)=\Delta_{0}(a, b)+\frac{c_{-}(b)}{\sqrt{|1+b-2 a|}},
$$

for some function $c_{-}$. Notice that $\Delta$ and $\Delta_{0}$ are by definition (27) and (11) continuous on $(0,+\infty)^{2}$. By letting $a$ goes to $(1+b) / 2$, we deduce that $c_{+}=c_{-}=0$. This proves the result.

Recall the definition of $I$ given in (13). We set for $a \geq 0, b \geq 0$ :

$$
J(a, b)=\int_{0}^{\infty} \frac{d \theta}{\theta+1}\left(\frac{\theta}{\sqrt{\theta^{2}+2 a \theta+b}}-\frac{\theta}{\theta+1}\right) .
$$

Lemma 5.5. For $a \geq 0, b \geq 0$, we have $J(a, b)=I(a, b)$.

Proof. We first notice that:

$$
\int_{0}^{\infty} \frac{\theta d \theta}{\left(\theta^{2}+2 a \theta+b\right)^{3 / 2}}=\frac{1}{\sqrt{b}+a} \quad \text { and } \quad \int_{0}^{\infty} \frac{d \theta}{\left(\theta^{2}+2 a \theta+b\right)^{3 / 2}}=\frac{1}{\sqrt{b}(\sqrt{b}+a)}
$$

For $a \geq 0, b \geq 0$ and $a+b>0$, we set:

$$
\Delta(a, b)=\int_{0}^{\infty} \frac{d \theta}{(\theta+1) \sqrt{\theta^{2}+2 a \theta+b}}
$$

We have:

$$
\begin{aligned}
-\partial_{b} J(a, b) & =\frac{1}{2} \int_{0}^{\infty} \frac{\theta d \theta}{(\theta+1)\left(\theta^{2}+2 a \theta+b\right)^{3 / 2}} \\
& =\frac{1}{2(1+b-2 a)} \int_{0}^{\infty} \frac{d \theta}{\sqrt{\theta^{2}+2 a \theta+b}}\left[\frac{\theta+b}{\theta^{2}+2 a \theta+b}-\frac{1}{\theta+1}\right] \\
& =-\frac{\Delta(a, b)}{2(1+b-2 a)}+\frac{1}{2(1+b-2 a)}\left[\frac{1}{\sqrt{b}+a}+\frac{b}{\sqrt{b}(\sqrt{b}+a)}\right] \\
& =-\frac{\Delta(a, b)}{2(1+b-2 a)}+\frac{1}{2(1+b-2 a)} \frac{\sqrt{b}+1}{\sqrt{b}+a} .
\end{aligned}
$$

We also have:

$$
\begin{aligned}
-\partial_{a} J(a, b) & =\int_{0}^{\infty} \frac{\theta^{2} d \theta}{(\theta+1)\left(\theta^{2}+2 a \theta+b\right)^{3 / 2}} \\
& =\frac{1}{1+b-2 a} \int_{0}^{\infty} \frac{d \theta}{\sqrt{\theta^{2}+2 a \theta+b}}\left[\frac{(b-2 a) \theta-b}{\theta^{2}+2 a \theta+b}+\frac{1}{\theta+1}\right] \\
& =\frac{\Delta(a, b)}{1+b-2 a}+\frac{1}{1+b-2 a}\left[\frac{b-2 a}{\sqrt{b}+a}-\frac{b}{\sqrt{b}(\sqrt{b}+a)}\right] \\
& =\frac{\Delta(a, b)}{1+b-2 a}+\frac{1}{1+b-2 a} \frac{b-\sqrt{b}-2 a}{\sqrt{b}+a} .
\end{aligned}
$$

After computing $\partial_{a} \Delta(a, b)$, we deduce from (29) that, for $a+b>0$ :

$$
J(a, b)=-\Delta(a, b)-\log (\sqrt{b}+a)+g(b),
$$


for some function $g$. Then computing $\partial_{b} J(a, b)$, we get using (28) that $g(b)$ is a constant $c$. Eventually, on the one hand taking $a=\sqrt{b}=1$, we get:

$$
J(1,1)=\int_{0}^{\infty} \frac{d \theta}{\theta+1}\left[\frac{\theta}{\theta+1}-\frac{\theta}{\theta+1}\right]=0 .
$$

On the other hand, we have:

$$
J(1,1)=-\Delta(1,1)-\log (2)+c=-1-\log (2)+c .
$$

This gives $c=1+\log (2)$. We get that:

$$
J(a, b)=-\Delta(a, b)-\log (\sqrt{b}+a)+1+\log (2) .
$$

That is $I=J$ for $a+b>0$. Then, we use the continuity of $I$ and $J$ to get $I=J$.

\section{REFERENCES}

[1] R. ABRAHAM and J.-F. DELMAS. Record process on the continuum random tree. arXiv:1107.3657, 2011.

[2] R. ABRAHAM and J.-F. DELMAS. A continuum tree-valued Markov process. Ann. of Probab. 40, 11671211, 2012.

[3] R. ABRAHAM, J.-F. DELMAS and H. HE. Pruning of CRT sub-trees. In preparation 2012.

[4] R. ABRAHAM, J.-F. DELMAS and G. VOISIN. Pruning a Lévy continuum random tree. Elec. J. of Probab. 15, 1429-1473, 2010.

[5] R. ABRAHAM and L. SERLET. Poisson snake and fragmentation. Elec. J. of Probab. 7, 1-15, 2002.

[6] D. ALDOUS. The continuum random tree I. Ann. of Probab. 19, 1-28, 1991.

[7] D. ALDOUS. The continuum random tree III. Ann. of Probab. 21, 248-289, 1993.

[8] D. ALDOUS and J. PITMAN. The standard additive coalescent. Ann. Probab., 26(4):1703-1726, 1998.

[9] N. BERESTYCKI. Recent progress in coalescent theory. Ensaios Matemáticos 16, 1-193, 2009.

[10] J. BERESTYCKI and N. BERESTYCKI and J. SCHWEINSBERG. Beta-coalescents and continuous stable random trees. Ann. Probab., 35(5): 1835-1887, 2007.

[11] J. BERTOIN and M. YOR. Exponential functionals of Lévy processes. Probability Surveys, 2: 191-212, 2005.

[12] M. BIRKNER, J. BLATH, M. CAPAldo, A. ETHERIDGE, M. MOEHLE, J. SCHWEINSBERG, A. WAKOLBINGER. Alpha-stable branching and Beta-coalescents. Elec. J. of Probab. 10, 303-325, 2005.

[13] E. BOLTHAUSEN and A.-S. SZNITMAN. On Ruelle's probability cascades and an abstract cavity method. Comm. Math. Phys. 197, 247-276, 1998.

[14] J.-F. DELMAS and Y.-T. CHEN. Smaller population size at the MRCA time for stationary branching processes. Ann. of Probab. to appear.

[15] T. DUQUESNE and J.-F. LE GALL. Random trees, Lévy processes and spatial branching processes. Astérisque, Vol. 281, 2002.

[16] S. EVANS. Probability and real trees. Ecole d'été de probabilités de Saint-Flour 2005, Lecture Notes in Math. 1920, Springer, 2008.

[17] C. FOUCARD and O. HENARD. Continuous-state branching processes with immigration and generalized Fleming-Viot processes with immigration. arXiv:1203.2494, 2012.

[18] C. GOLDSCHMIDT and J. MARTIN. Random recursive trees and the Bolthausen-Sznitman coalescent. Elec. J. of Probab. 10, 718-745, 2005.

[19] A. GNEDIN and A. IKSANOV and A. MARYNYCH. Lambda-coalescents with dust component Preprint arXiv:1102.1146v1, 2011.

[20] P. HOSCHEIT. Fluctuations for the number of records on random binary trees. In preparation. 2012.

[21] A. IKSANOV and M. MÖHLE. On the number of jumps of random walks with a barrier. Adv. Appl. Probab. 40, 206-228, 2008.

[22] S. JANSON. Random cutting and records in deterministic and random trees. Random Structures Algorithms 29, 139-179, 2006.

[23] J.F.C. KINGMAN. The coalescent. Stoch. Proc. and Appl. 13, 235-248, 1982.

[24] J.F. LE GALL. Random real trees. Ann. Fac. Sci. Toulouse Math. 15, 35-62, 2006.

[25] J. PITMAN. Coalescents with multiple collisions. Ann. Probab. 27, 1870-1902, 1999. 
[26] J. PITMAN. Combinatorial stochastic processes. Ecole d'été de probabilités de Saint-Flour 2002, Lectures Notes in Math., 1875, Springer, 2006.

Romain Abraham, Laboratoire MAPMO, CNRS, UMR 6628, FÉdération Denis Poisson, FR 2964, Université d'Orléans, B.P. 6759, 45067 Orléans cedex 2, France.

E-mail address: romain.abraham@univ-orleans.fr

Jean-François Delmas, Université Paris-Est, École des Ponts, Cermics, 6-8 av. Blaise PasCal, Champs-sur-Marne, 77455 Marne La Vallée, France.

E-mail address: delmas@cermics.enpc.fr 